\title{
5. ACERCA DEL TRATAMIENTO DE LEMNACEAE EN FLORA IBERICA
}

\section{Antonio GALÁN DE MERA y Santiago CASTROVIEJO}

Como es bien sabido, para la delimitación y ordenación de las familias de angiospermas, en Flora iberica, se decidió desde un principio seguir el sistema de clasificación propuesto por Stebbins (1974, Flowering plants - Evolution above the species level). Hoy, por mera coherencia del conjunto de la obra, seguimos manteniendo el mismo criterio a pesar de que somos conscientes de los cambios propuestos a la luz de la enorme cantidad de información que aflora con los estudios moleculares y de nuevas interpretaciones hechas de los datos morfológicos.

En este caso están, por ejemplo, las Lemnáceas, ya que algunos autores [Govaerts \& Frodin, World Checklist Araceae: 3 (2002); Keating in Ann. Missouri Bot. Gard. 91: 490 (2004), etc.], basándose fundamentalmente en la presencia de una diminuta espata que bordea las flores, las incluyen dentro de las Aráceas. Reconocemos las Lemnáceas, entre otros caracteres, por sus pequeñas frondes, en cuyo nudo confluyen nervios más o menos visibles, raíces y estípites, y por la espata que generalmente rodea a sus flores.

Para la preparación de la síntesis de los géneros nos hemos basado fundamentalmente en los trabajos taxonómicos de Den Hartog \& Van der Plas [Blumea 18: 355-368 (1970)], Kandeler [Aquatic Bot. 1: 365-376 (1975)], Landolt [Veröff. Geobot. Inst. Rübel Zürich 71: 1-563 (1986) y Ber. Geobot. Inst. E.T.H. Stiftung Rübel 60: 137-151 (1994)] y, sobre todo, en la síntesis de Landolt para la flora de
América del Norte [Fl. N America 22 (2000)], que comprende latitudes semejantes a las ibéricas.

Como consecuencia del reestudio de todo el material disponible, proponemos ahora el siguiente esquema taxonómico para la familia en la Península:

\section{Lemnoideae}

\section{Lemna L.}

1. L. trisulca L., Sp. P1.: 970 (1753)

2. L. minor L., Sp. P1.: 970 (1753)

3. L. gibba L., Sp. P1.: 970 (1753)

4. L. minuta Kunth in Humb., Bonpl. \& Kunth, Nov. Gen. Sp. 1: 372 (1815)

[L. minuscula Herter in Rev. Sudamer. Bot. 9: 185 (1954)]

5. L. valdiviana Phil. in Linnaea 33: 239 (1864)

2. Spirodela Schleid.

1. S. polyrrhiza (L.) Schleid. in Linnaea 13: 392 (1839)

Lemna polyrrhiza L., Sp. P1.: 970 (1753) [basión.]

2. S. punctata (G. Meyer) C.H. Thompson in I Rep. (Annual) Missouri Bot. Gard. 9: 28 (1898)

Lemna punctata G. Meyer, Prim. Fl. Esseq.: 262 (1818) [basión.]

Wolffioideae Engl.

3. Wolffia Horkel [nom. cons.]

1. W. arrhiza (L.) Horkel ex Wimm., F1. Schles.: 140 (1857)

Lemna arrhiza L., Mant. P1. 2: 294 (1771) [basión.] 
A los datos ya conocidos, podemos añadir:

Lemna minuta Kunth in Humb., Bonpl. \& Kunth, Nov. Gen. Sp. 1: 372 (1815)

Port. DOURO LITORAL: Porto, S. Mamede de Infesta, numa preza, 15-XI-1941, Joaquín Castro (PO 3743).

Se sabía desde hace tiempo [Jovet \& JovetAst, Bull. Centr. Études Rech. Sci. 6(1): 57 (1966); Aizpuru et al., Claves Fl. País Vasco: 603 (1999)] de su presencia en el País Vasco francés. Más recientemente, Aizpuru et al. la citaron de los arrozales de Tudela (Navarra) [Munibe 51: 49 (2001)]. En este otoño, hemos vuelto a buscarla a la misma localidad, acompañados por el propio Iñaki Aizpuru, pero no la hemos encontrado. El pliego testigo de la recolección parece haberse extraviado en ARAN. Así pues, el código correspondiente a Navarra aparecerá entre paréntesis.

Muy recientemente, al estudiar los materiales del herbario de Oporto (PO) hemos encontrado la muestra que confirma por primera vez su presencia en Portugal.

Lemna valdiviana Phil. in Linnaea 33: 239 (1864)

Port. BEIRA LITORAL: Montemor-oVelho, El Taipal, 24-V-1995, S. Cirujano \& $P$. García Murillo (MA 624161).

Al parecer, es la primera cita peninsular de esta planta americana.

Spirodela punctata (G. Meyer) C.H. Thompson in Rep. (Annual) Missouri Bot. Gard. 9: 28 (1898)

Port. DOURO LITORAL: Porto, Lordelo, num rego de agua, pr. do ermiterio, 21-VII-1969, G. Costa (PO27299).

Segunda cita para la Península; la primera, [Galán de Mera et al., Acta Bot. Malacitana 30:???(2005)] se basa en pliegos procedentes de Huelva (MA 681551, MGC 51204).

Wolffia arrhiza (L.) Horkel ex Wimm., Fl. Schles.: 140 (1857)
Port. BAIXO ALENTEJO: Aguas de Moura, posta experimental do Vale de Sado, 22-VIII1955, J. de Vasconcellos (LISI 55227); Alcácer do Sal, Herdade da comporta (do lado do estuário), 24-III-1982, M. do Carmo Fernandez (LISI). Los ejemplares de $W$. arrhiza aparecen mezclados con otros de L. gibba.

Sabíamos que en Portugal la planta era conocida de Estremadura [E: Palmela, Águas de Monsanas valas dos campos de arroz do Instituto de Malariologia, VIII-1953, E.J. Mendes \& Pedro Frra. Marques (LISU P56380)], Beira Litoral [Pereira Coutinho, Fl. Portugal: 135 (1939)] y del Ribatejo [R: Benavente, Campo de Tiro de Alcochete, 29SNC1292, $30 \mathrm{~m}$, charcas permanentes oligotróficas, VII-1999, Miravent \& Draper (LISU 171350)]. En España ha sido herborizada en Huelva y Cádiz [H: Punta Umbría, laguna del Portil, VII-1979, S. Silvestre (SEV 96961); Doñana, E1 Hondón, 29SQA2793, 19VII-2001, E. Sánchez Gullón, D. Cobos \& P.G. Murillo (MGC 51228); Ca: Algeciras, E1 Rinconcillo, cultivos, 28-VI-1979, J. Rivera \& S. Silvestre (SEV 96960)].

Aceptado para su publicación en octubre de 2005

Dirección de los autores. A. GALÁN DE MERA: Departamento de CC Ambientales y Recursos Naturales, Laboratorio de Botánica, Universidad San Pablo-CEU, Apartado 67, E-28660 Boadilla del Monte, Madrid (agalmer@ceu.es); S. CASTROVIEJO: Real Jardín Botánico (CSIC), Plaza de Murillo 2, E-28014 Madrid (castroviejo@ma-rjb.csic.es). 